DOI: https://doi.org/10.46296/yc.v4i7edesp.0067

\title{
ESTRÉS LABORAL EN DOCENTES DE EDUCACIÓN BÁSICA POR EL CAMBIO DE MODALIDAD DE ESTUDIO PRESENCIAL A VIRTUAL
}

\section{WORK STRESS IN BASIC EDUCATION TEACHERS DUE TO THE CHANGE OF THE MODALITY OF STUDYING IN PERSONAL TO VIRTUAL}

\author{
Párraga-Párraga Kléber Leonardo ${ }^{1 *}$; Escobar-Delgado Gustavo Rafael ${ }^{2}$ \\ ${ }^{1}$ Universidad Técnica de Manabí, Carrera de Psicología Clínica. Portoviejo - Ecuador. \\ ${ }^{2}$ Docente de la Universidad Técnica de Manabí, Facultad de Ciencias Humanísticas y \\ Sociales. Portoviejo - Ecuador.
}

*Correo: leoparraga21@gmail.com

\begin{abstract}
Resumen
La emergencia sanitaria planetaria, causada por la pandemia del Covid-19, ha llevado a todos los gobiernos a cuestionarse si realmente están preparados para una perentoriedad de tal magnitud. Una de las tantas áreas afectadas es la educación. Específicamente en Ecuador, el cambio de modalidad de clases resultó un apremiante reto para todos los actores directos e indirectos, especialmente alumnos y docentes. El nuevo desempeño laboral, caracterizado por el cambio de modalidad de clases lleva a determinar el desgaste tanto físico como mental de los docentes. Por ello, es necesario responder las siguientes cuestiones: ¿Existe consecuencias en la salud mental de los trabajadores? ¿Cómo evitar fuertes impactos emocionales? ¿El cambio de modalidad de clases aumenta la probabilidad de sufrir estrés laboral en los docentes? Para responder estas cuestiones se llevó a cabo un estudio de carácter cuantitativo con perspectiva correlacional. La investigación tomó una muestra de 20 docentes de educación general básica de cuatro entidades educativas de la parroquia Abdón Calderón del cantón Portoviejo. Se aplicaron dos baterías de test: El cuestionario de problemas psicosomáticos de Hock y la escala de satisfacción laboral de Warr. Se evidenció que el $90 \%$ de la muestra seleccionada padece de estrés laboral en diferentes niveles. Además, se consideraron variables de evaluación como edad, sexo, estado civil y número de hijos, comprobándose que las mismas coadyuvan para la presencia del estrés en los docentes de educación general básica.
\end{abstract}

Palabras clave: estrés, docentes, educación, pandemia, Covid-19.

\begin{abstract}
The planetary health emergency, caused by the Covid-19 pandemic, has led all governments to question whether they are really prepared for a peremptory situation of such magnitude. One of the many areas affected is education. Specifically in Ecuador, the change in classrooms was a pressing challenge for all direct and indirect actors, especially students and teachers. The new work performance, characterized by the change in classrooms, leads to the physical and mental wear and tear of teachers. Therefore, it is necessary to answer the following questions: Are there consequences in the mental health of workers? How to avoid strong emotional impacts? Does the change of classrooms increase the probability of suffering work stress in teachers? To answer these questions, a quantitative study with a correlational perspective was carried out. The research took a sample of 20 teachers of basic general education from four educational entities in the parish of Abdón Calderón in the canton of Portoviejo. Two test batteries were applied: Hock's psychosomatic problems questionnaire and Warr's job satisfaction scale. It was shown that $90 \%$ of the selected sample suffers from labor stress at different levels. In addition, evaluation variables such as age, sex, marital status and number of children were considered, proving that they contribute to the presence of stress in teachers of basic general education.
\end{abstract}

Keywords: stress, teachers, education, pandemic, Covid-19.

Información del manuscrito:

Fecha de recepción: 17 de septiembre de 2020.

Fecha de aceptación: 12 de noviembre de 2020.

Fecha de publicación: 16 de noviembre de 2020. 


\section{Introducción}

La situación actual a la que está expuesta el mundo debido a la pandemia del Covid-19 ha llevado a los gobiernos de todos los países a tomar acciones específicas para el funcionamiento efectivo de sus instituciones en general. Muchas medidas siguen debatiéndose, dadas las escasas referenciaciones históricas frente a devastadores fenómenos como la pandemia por coronavirus. Siendo un suceso muy complejo, para los gobiernos no es fácil determinar la pertinencia de una medida que permita brindar servicios adecuados a sus ciudadanos, sin descuidar la salud física y mental.

Entre las estrategias adoptadas para que las instituciones tanto públicas como privadas sigan funcionando, el teletrabajo se erige como el método más usado alrededor del mundo; sin embargo, como es bien sabido, la actividad telemática tiene muchas limitaciones que impiden optimizar en el $100 \%$ los servicios a la comunidad y por ende garantizar la interacción dinámica y efectiva propia de los sistemas productivos.

Uno de los sectores más sensibles, junto a la salud, sin duda ha sido la educación; pues, no solamente se forzó el replanteamiento y modificación de los calendarios estudiantiles, sino que, sobre la marcha, se suscitaron acciones didáctico-pedagógicas no convencionales, pero que buscaban y buscan la aprehensión racional de la realidad y la potencial intervención en aras del mejoramiento de la calidad de vida. Así, la crisis sanitaria se convirtió en una barrera desconcertante e inteligible que hay que enfrentar, pensando siempre en la adopción de estrategias que potencien el andamiaje político integral de las sociedades.

Específicamente, en Latinoamérica, el desempeño académico virtual ha sido el método que ha buscado mantener la educación en términos eficientistas. Sin embargo, con ello se pudo poner en evidencia ciertos problemas de la comunidad educativa de la región. Tal y como lo menciona García (2020): "La suspensión de las clases presenciales provocada por la pandemia de coronavirus ha exigido un replanteamiento de las tareas de enseñanza-aprendizaje y del trabajo 
del alumnado inimaginables para cualquiera de nosotros" (p. 13).

Falta de experiencia tecnológica, plataformas educativas ineficientes, población docente envejecida, alarmante ausencia de conectividad a internet en el sector urbano y más aún en las zonas rurales, entre otros inconvenientes, ha demostrado que ciertos países no están listos para la necesaria y asfixiante revolución tecnológica que representa la situación mundial actual, y nuestro país no es la excepción.

Dichos factores no solo han afectado la calidad de la educación y el desarrollo de las capacidades de los estudiantes; también han llevado a los docentes a enfrentar un apremiante reto tecnológico para el que quizás no estaban preparados, independientemente de las repercusiones en sectores estratégicos directamente vinculados con el desarrollo industrial y financiero de una nación. El docente actual atraviesa la imperiosa necesidad de auto revisar no solamente la dimensión tecnológica de la educación, sino también, la científica y humanística. Al hacerlo, adoptará una actitud profiláctica a través la cual evitaría perturbaciones tanto de la salud física como mental. Tal y como menciona Barreto y Piamonte (2020) los docentes en esta situación tienden a padecer de síndrome de burnout, también conocido como síndrome del trabajador quemado ( $p$. 26).

El desgaste mental en los docentes no es nuevo, está presente en la comunidad educativa latinoamericana, debido, entre otros factores, a los bajos sueldos, intensos horarios de trabajo, desgaste cognitivo-afectivo y precario ambiente laboral en el que deben manejarse. No obstante, las condiciones han cambiado, son brutales por la amenaza mortal de la pandemia. Por otro lado, Ayuso (2006), menciona que la indisciplina de los alumnos, falta de apoyo de los padres e incluso la mala administración educativa son factores que llevan a la baja de personal docente por enfermedades mentales (p. 2).

Entre las enfermedades mentales más recurrentes en la comunidad docente está el estrés laboral (Oliveira \& Kieling, 2018). Para Vidal (2019). El estrés laboral es un desequilibrio percibido entre las 
demandas profesionales y la capacidad de la persona para llevarlas a cabo (p. 36). Esta definición lleva a suponer que un factor latente, desencadenante del estrés laboral, está asociado funcionalmente con la esfera cognitiva-afectiva; pues, podría ocurrir una deficiente autopercepción que conllevaría a un sentimiento de ineptitud, en el docente, para el desempeño efectivo.

Por su parte, la Organización Mundial de la Salud (OMS - 2004) proporciona otra definición del estrés laboral: "el estrés laboral es la reacción que puede tener el individuo ante las exigencias $y$ presiones laborales que no se ajustan a sus conocimientos y capacidades, y que ponen a prueba su capacidad para afrontar la situación" (p. 3). Para la OMS, el estrés laboral se presenta como consecuencia de una sobre exigencia en la carga laboral, que es lo que, en estos momentos, el personal docente está padeciendo como consecuencia del cambio de modalidad de estudio.

En Ecuador, se habla de una crisis educativa debido a la falta de internet en los hogares 0 de artefactos tecnológicos como computadoras o laptops. De acuerdo con el Instituto Nacional de Estadísticas y Censos (INEC) $70 \%$ de estudiantes tiene dificultad en el acceso a la educación en línea. (2018, pág. 27), estos datos significan que la población de estudiantes ecuatorianos, no estaban listos para un cambio de modalidad tan abrupto como el ocurrido a causa de la pandemia del Covid-19.

Dichos datos no solo reflejan incapacidad de los estudiantes a acceder a las clases virtuales de manera óptima, esto también afecta a los docentes de educación básica en general, la población de docentes envejecida, sumado al analfabetismo tecnológico que existe en Ecuador (Instituto Nacional de Estadisticas y Censos, 2018). Así, un nuevo escenario académico se abre a los docentes y a los estudiantes; por lo que, surgen nuevos factores de predisposición para el estrés laboral.

A lo expresado, se agrega el atraso en los haberes económicos de los docentes fiscales ecuatorianos, justificado, según las autoridades financieras, por la falta de recursos 
económicos que ha traído la emergencia sanitaria. Habiendo cerca de 215255 profesores fiscales en el país, cerca del $70 \%$ de ellos ha tenido inconvenientes al momento de cobrar sus salarios (García A. , 2020), este escenario sería el promotor del agotamiento físico y mental, llevando a los docentes ecuatorianos a sufrir de estrés laboral.

En estas condiciones, el delicado rol que actualmente desempeña el docente, al afectar su condición mental, también afectaría la calidad de los aprendizajes de los estudiantes. Esta problemática incrementa el riesgo de desarrollar descalabros mentales en educadores de escuelas primarias 0 básicas. Lo expresado se refleja en los estudios de Mascaró (2016), quien menciona que en comparación a los educadores de nivel secundario o terciario su salario es reducido ( $p$. 46). Se deduce que los educadores de nivel primario son mayormente propensos a padecer de estrés laboral y de contar con insatisfacción laboral.

En virtud de lo mencionado, la presente investigación está dirigida a establecer la posible presencia de estrés laboral en docentes de educación primaria, considerando que, como se ha descrito, este segmento social se encuentra actualmente en permanente presión laboral, motivada por la transición de modalidad presencial a virtual, dada la emergencia sanitaria por coronavirus.

La investigación se llevó a cabo con docentes fiscales de educación general básica de la parroquia Abdón Calderón, perteneciente al cantón Portoviejo, quienes constituyen unidades básicas de investigación para caracterizar el desempeño laboral propio de la educación telemática, en tiempos de pandemia.

\section{Metodología}

En el presente trabajo investigativo se utilizó una investigación de carácter cuantitativa con perspectiva correlacional. La metodología empleada permitió estudiar la correlación entre las variables y los resultados de la batería del test. Se utilizó el Cuestionario de Problemas Psicosomáticos (CPP) de Hock para establecer los niveles de estrés presentes en los docentes. Esta 
batería de test permitió evaluar los niveles de estrés laboral de manera rápida y eficiente mediante la frecuencia de aparición de síntomas psicosomáticos (Hock, 1988).

Por otra parte, y como adicional a la investigación central, se aplicó la Escala General de Satisfacción Laboral (Warr, Wall, \& Cook, 1979), la cual, al igual que el CPP es auto cumplimentados, lo que facilitó la aplicación de los mismos dada la situación de confinamiento que se atravesó al momento de la realización de la presente investigación. Dicha escala permitió correlacionar los datos sobre estrés laboral en relación con la satisfacción laboral, lo cual llevó a establecer variables adicionales a la investigación.

Los instrumentos fueron aplicados a veinte docentes de cuatro instituciones de educación general básica de la parroquia Abdón Calderón en la ciudad de Portoviejo. Como muestra se tomó en cuenta cinco docentes de cada escuela incluyendo entre ellos al rector de cada institución. Para la aplicación del test, se utilizó un formulario en línea, dado que por motivos de confinamiento se imposibilitó la aplicación de manera presencial. Como agregado al formulario, se indagó variables como: sexo, estado civil, edad y número de hijos.

\section{Resultados}

\subsection{Niveles de estrés laboral en docentes de educación primaria}

La muestra seleccionada está distribuida por sexo, dando una diferenciación mujeres-hombres de 15-5 respectivamente, demostrando una mayor presencia de sexo femenino con el $75 \%$ tal y como se evidencian en la Figura 1, esto a su vez se relaciona con los datos demostrados en el informe realizado por el Instituto Nacional de Evaluación Educativa (INEE), donde se evidenció que la presencia de mujeres en la docencia del cantón de Manabí superó el 70\% (2018, p. 101). Por otro lado, el rango de edad predominante es el comprendido entre los 31 y 40 años, estos también muestran relación con los datos del INEE (p. 105). 
Figura 1. Distribución de la Población por sexo, y estado civil.

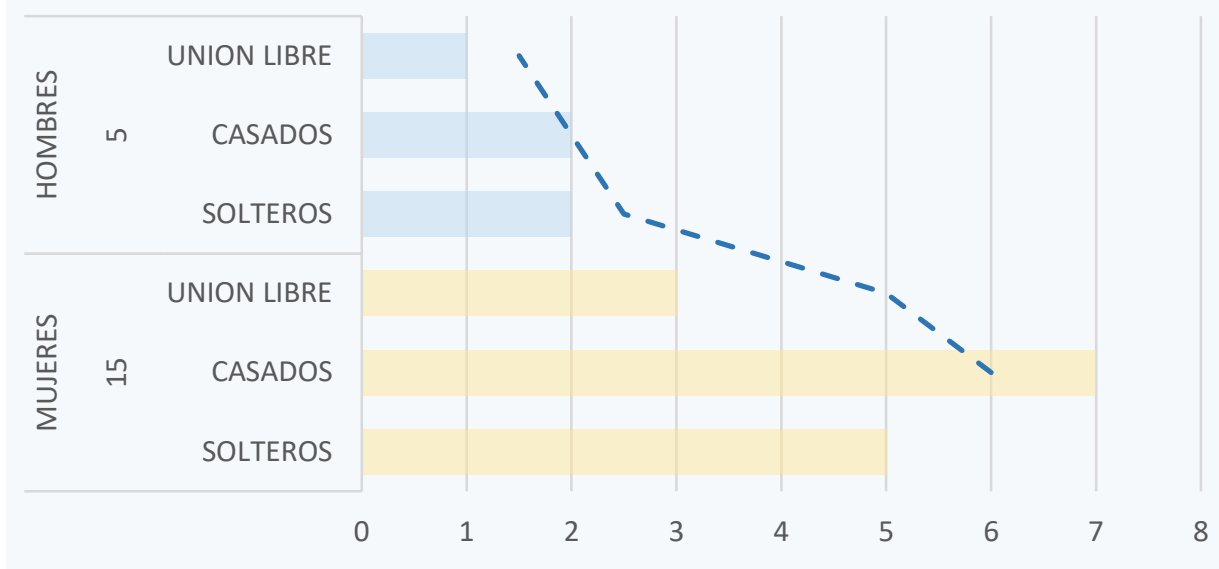

Elaborado por: Autores (2020).

En la Figura 2, se puede observar los niveles de estrés laboral que presenta la población evaluada. Entre los datos a destacar, se encontró que el $90 \%$ de la población cuenta con algún nivel de estrés laboral, esto demuestra que el cambio abrupto de modalidad presencial a teletrabajo de clases ha incidido en la salud mental de los docentes.

Tanto en el sexo masculino como en el femenino se detectó un $35 \%$ de casos tanto de estrés leve como estrés medio, los cuales corresponden al nivel de estrés con mayor incidencia. Hock describió estos niveles de estrés como de alarma ya que, si la situación estresante se prolonga, puede romper el equilibrio entre lo laboral y lo personal (Instituto Mexicano del Seguro Social, 2019).
Se puede observar la presencia de casos de niveles de estrés tanto altos como graves con un $20 \%$, además de que dicha muestra corresponde a la población femenina, esto lleva a suponer que la población femenina es más propensa a la aparición de niveles altos de estrés laboral, tal y como se evidenció en la investigación elaborada por Velásquez y Escobar (2020, p. 7). Es menester mencionar que la presencia de estrés laboral en niveles altos es un indicador de gravedad a tener en cuenta, esto a su vez refleja que el cambio de modalidad de clases de presencial a virtual ha complicado no solo la carga laboral de los docentes, sino que también ha desencadenado desgaste físico y mental. 
Figura 2. Niveles de estrés laboral en docentes de educación primaria.

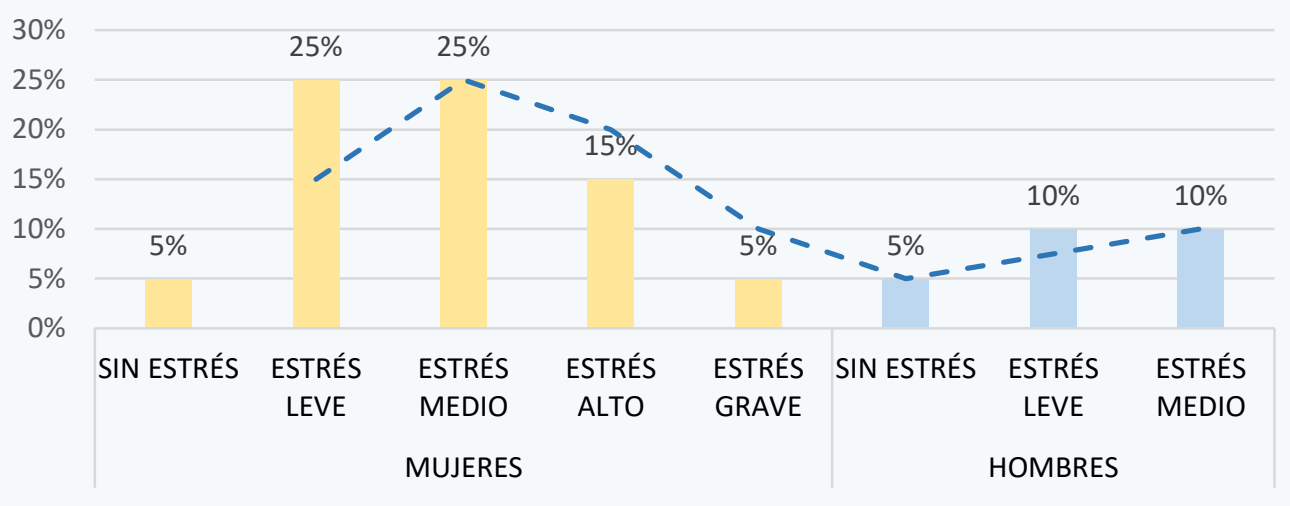

Elaborado por: Autores (2020).

En cuanto a la relación del estrés laboral con las variables planteadas, se encontró en la Figura 3 que la población casada tiende a mostrar mayor incidencia en niveles de estrés laboral elevados, resultados que mostraron relación con los encontrados por Velásquez y Escobar (2020, p. 11), quienes mencionaron que la población casada es más propensa a sufrir de estrés laboral.
La Figura 3 permite evidenciar que la población con hijos mostró mayor predisposición a padecer de estrés laboral ya que el $90 \%$ de la población con hijos padeció de algún tipo de nivel de estrés laboral, demostrado en la investigación de Ticona (2018), el contar con hijos es un factor predominante al momento de evaluar estrés laboral, además de que suelen mostrar incidencia en niveles de estrés elevados (p. 79).

Figura 3. Estrés laboral en relación con la variable Estado Civil y número de hijos

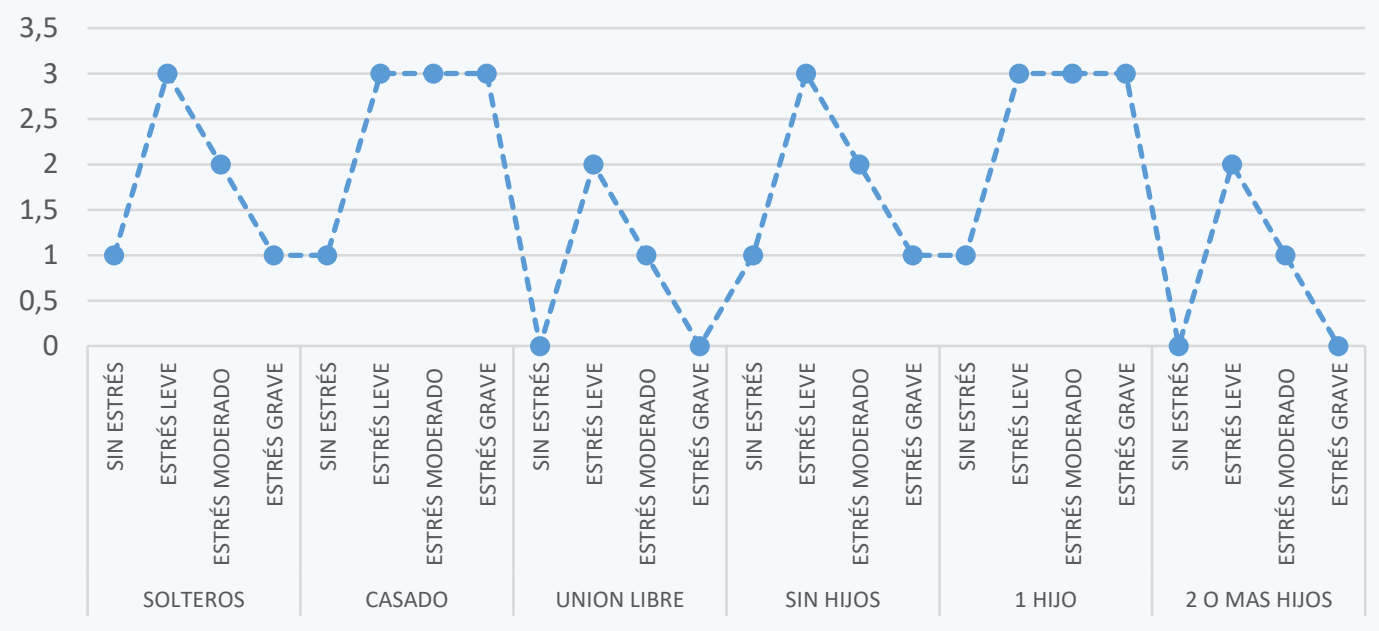

Elaborado por: Autores (2020). 


\subsection{Escalas de Satisfacción}

\section{Laboral en docentes de educación} primaria.

La Figura 4 determina el nivel de satisfacción laboral que se presentó en la población docente. Como dato a destacar se encontró que el $50 \%$ de los evaluados se encontraron categorizados como insatisfechos en sus labores, frente al $20 \%$ que se ubicó como satisfechos laboralmente. Entre los factores que pudieron provocar este porcentaje, se encuentran la emergencia satinaría, el confinamiento, la falta de pagos a los docentes fiscales, entre otros.

Una de las causas principales de la alta insatisfacción laboral en los docentes, puede ser atribuida al cambio de metodología en la planificación estudiantil para la transición de modalidad de clases. Como lo explica García y Taberna (2020), se tuvo que realizar un cambio de modalidad "Express" para poder dar cumplimiento al calendario estudiantil en la mayoría de los países (p. 184).

Cabe mencionar que estos resultados se pueden ligar a los hallados por Alves, López y Precioso (2020) quienes mencionan que en los docentes la percepción de bienestar ha disminuido a partir de la pandemia (p. 203). Esto puede relacionarse con la insatisfacción laboral causada por varias variables como la incertidumbre del futuro profesional de los docentes.

Figura 4. Satisfacción laboral en la población de docentes de educación primaria.

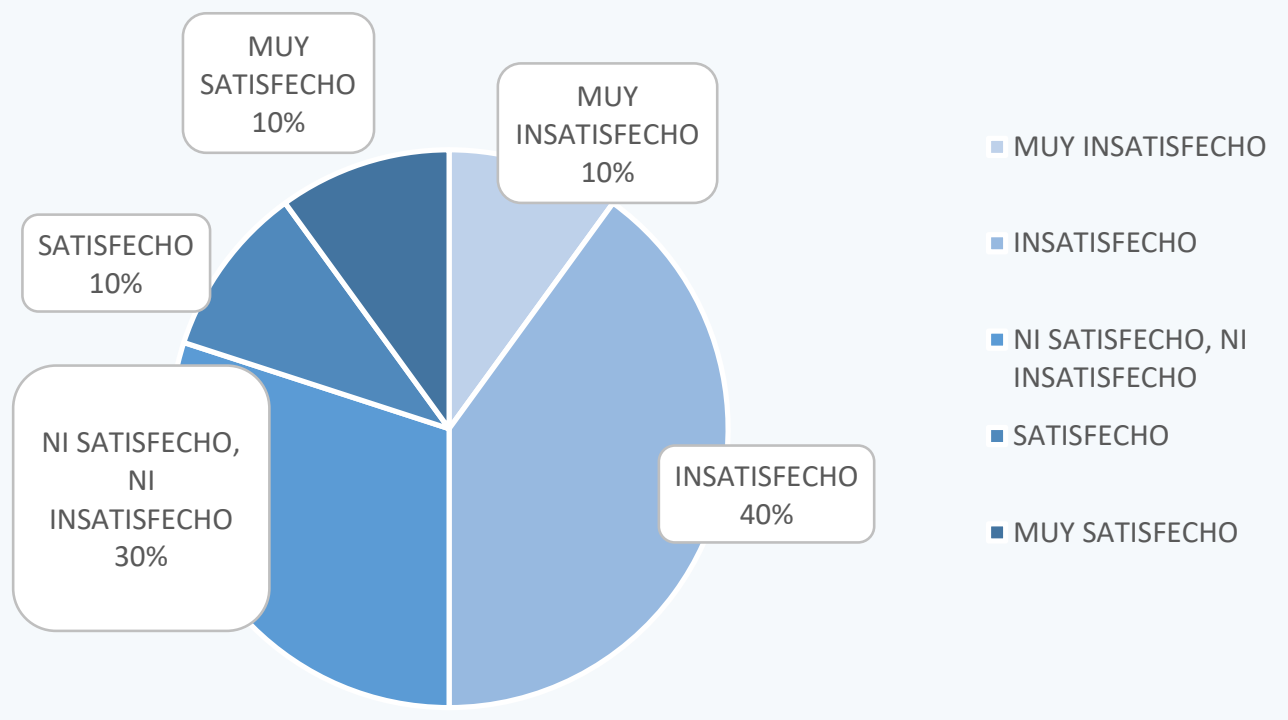

Elaborado por: Autores (2020). 
En cuanto a la relación entre estrés laboral y satisfacción laboral, se puede observar en la Tabla 1 que los valores relacionados con estrés tanto medio, alto y grave se encuentran ubicados en niveles de insatisfacción laboral. Significa esto que el estrés laboral está relacionado con el nivel de satisfacción laboral, lo cual se mostró más evidente en la Figura 5, donde el estrés alto y grave está direccionados hacia la insatisfacción laboral.

Tabla 1. Relación estrés laboral y escalas de satisfacción laboral.

\begin{tabular}{cccccc}
\hline & $\begin{array}{l}\text { Sin } \\
\text { Estrés }\end{array}$ & $\begin{array}{l}\text { Estrés } \\
\text { Leve }\end{array}$ & $\begin{array}{c}\text { Estrés } \\
\text { Medio }\end{array}$ & $\begin{array}{l}\text { Estrés } \\
\text { Alto }\end{array}$ & $\begin{array}{l}\text { Estrés } \\
\text { Grave }\end{array}$ \\
\hline Muy Satisfecho & & & & & \\
Satisfecho & 2,00 & & & & \\
Indiferente & & 1,00 & 1,00 & & 1,00 \\
Insatisfecho & & 4,00 & 2,00 & & \\
Muy Insatisfecho & & 2,00 & 4,00 & 1,00 & 2,00 \\
\hline
\end{tabular}

Elaborado por: Autores (2020).

Figura 5. Relación Estrés Laboral y Satisfacción Laboral

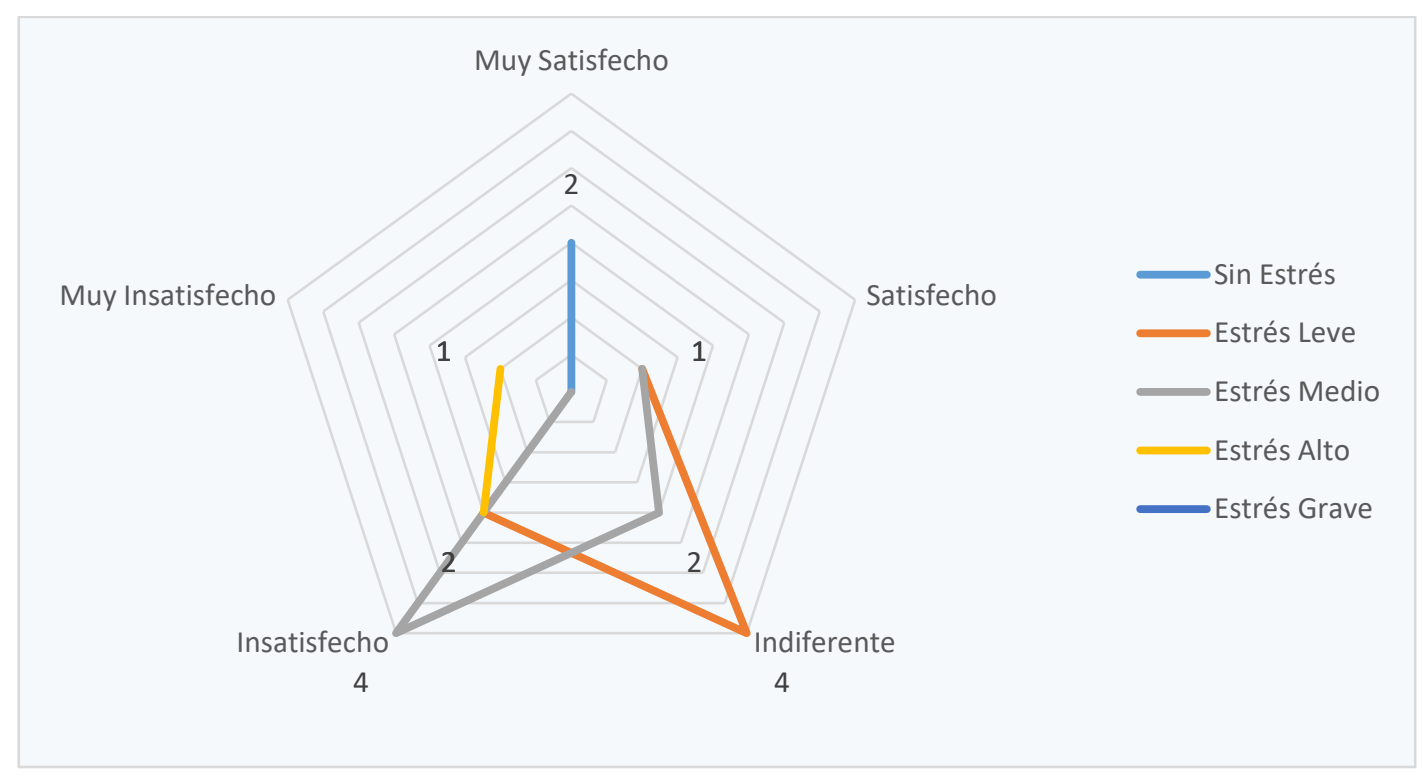

Elaborado por: Autores (2020). 


\section{Conclusiones}

En el lapso de la investigación, y por la emergencia sanitaria promovida por la pandemia del Covid-19, prácticamente todas las instituciones alrededor del mundo generaron medios y métodos de funcionamiento para minimizar la probabilidad de contagios, esto llevó a las unidades educativas a cambiar su modalidad de trabajo presencia a clases virtuales, ocasionando grandes y complejos retos para docentes, alumnos, personal administrativo y padres de familia.

Gracias a los datos recolectados se pudo llegar a la conclusión de que diversos factores ocasionaron malestar en la salud mental de los docentes de educación general básica, construyéndose un porcentaje de alrededor del $90 \%$ de docentes con algún tipo de nivel de estrés; siendo el estrés leve y el estrés medio los niveles predominantes con el 35\% de incidencia en la salud mental. El 10\% de la población evaluada no presenta signos de algún tipo o nivel de estrés laboral.

Se pudo evidenciar que la población femenina es más proclive a padecer de estrés laboral en niveles elevados; este aserto se relaciona con variables como el estar casado o tener hijos; así se establece un perfil de docentes de educación general básica propensos a padecer estrés laboral. Los datos estadísticos y la información recabada constituyen aspectos relevantes para tener en cuenta para la toma de medidas que fomenten el aprendizaje y salud mental funcionales.

La investigación permitió evidenciar que la satisfacción laboral está estrechamente relacionada con el estrés laboral, dado que los datos permitieron mostrar que, el estar insatisfecho laboralmente es un indicador del padecimiento de estrés laboral en los docentes. Se infiere que, en muchos de los casos esta insatisfacción es resultado de factores como el incumplimiento de los pagos económicos mensuales, la mala organización a causa del cambio de modalidad de clases, entre otros factores.

También, es necesario mencionar que los niveles de estrés e insatisfacción laboral encontrados pueden ser resultado de la abrumante situación que se atraviesa alrededor del mundo a 
causa de la pandemia por coronavirus. Ya que estudios como el de Muños, Molina, Ochoa, Sánchez y Esquivel (Muñoz, Molina, Ochoa, Sanchez, \& Esquibel, 2020), mostraron que la pandemia por COVID-19 incidió gravemente en la salud mental de la población.

\section{Bibliografía}

Alves, R., Lopes, T., \& Precioso, J. (2020). El bienestar de los docentes en tiempos de pandemia Covid-19: factores que explican. International Journal of Educational Research and Innovation(15), 203-217.

Ayuso, J. (2006). Profesión docente y estrés laboral: una aproximación a los conceptos de Estrés Laboral y Burnout. Revista Iberoamericana de Educación, 2-14. Obtenido de https://d1wqtxts1 xzle7.cloudfr ont.net/31741875/Profesion docente_y_estres_laboral.pdf ?1376871512=\&responsecontentdisposition=inline $\% 3 \mathrm{~B}+$ filena me\%3DProfesion_docente_y _estres_laboral.pdf\&Expires $=$ 1595092390\& Signature $=$ fof $Y$ PY4C4luGVXS6PHzJa6xJDA oV12bqfVsiJ8w

Barreto, M., \& Piamonte, J. (2020). Estrés laboral en comunidad docente. Universidad
Cooperativa de Colombia, 625. Obtenido de https://repository.ucc.edu.co/ bitstream/20.500.12494/1767 8/3/2020_estres_laboral.pdf

García, A. (2020). Dos gremios de docentes piden que se complete pago de sueldos. El comercio. Obtenido de https://www.elcomercio.com/ actualidad/gremios-docentessalarios-abrilcoronavirus.html

García, E. (2020). Reflexiones sobre la educación telemática en tiempos de coronavirus. AOSMA, 1(28), 13-19. Obtenido de: https://dialnet.unirioja.es/servl et/articulo?codigo $=7381628$

García, M., \& Taberna, J. (2020). Transición de la docencia presencial a la no presencial en la UPC durante la pandemia del COVID-19. Revista Internacional de Investigación e Innovación Educativa(15), 177-187. Obtenido de https://upo.es/revistas/index.p $\mathrm{hp} /$ IJERI/article/view/5015/45 37

Hock, R. (1988). Professional Burnout among Public School Teachers. Public Personnel Management, 17(2), 167-189.

Instituto Mexicano del Seguro Social. (2019). Test de Estrés Labodal. Obtenido de IMSS: http://www.imss.gob.mx/sites/ 
all/statics/salud/estreslaboral/

Test-Estres-Laboral.pdf

Instituto Nacional de Estadisticas y Censos. (2018). Ecuador en Cifras. Obtenido de INEC: https://www.ecuadorencifras. gob.ec/documentos/webinec/Estadisticas_Sociales/TI C/2018/201812_Principales_r esultados_TIC_Multiproposito .pdf

Instituto Nacional de Evaluación Educativa. (2018). La educación en Ecuador: logros alcanzados y nuevos desafíos. Resultados educativos 2017-2018. Quito: Instituto Nacional de Evaluación Educativa. Obtenido de https://www.evaluacion.gob.e c/wpcontent/uploads/downloads/2 019/02/CIE_ResultadosEduc ativos18_20190109.pdf

Mascaró, G. (2016). ¿Se estresa el profesorado? Estudio sobre el estrés laboral en el profesorado de un centro de Educación Primaria y Secundaria. Obtenido de Repositorio de la Universidad Internacional de La Rioja: https://reunir.unir.net/bitstrea m/handle/123456789/4001/M ASCARO $\% 20 G E N E S T A R \% 2$ C\%20CEL.pdf?sequence $=1 \&$ isAllowed $=y$

Muñoz, S., Molina , D., Ochoa, R., Sanchez, O., \& Esquibel, J. (2020). Estrés, respuestas emocionales, factores de riesgo, psicopatología y manejo del personal de salud durante la pandemia por COVID-19. Acta Pedriática de Mexico, 127-136.

Oliveira, A., \& Kieling, J. (2018). Educación superior y enfermedad del docente: un estudio en una universidad pública. Psicologia da Educação, 1-10. Obtenido de http://pepsic.bvsalud.org/sciel o.php?pid=S1414$69752018000100001 \&$ script $=$ sci_abstract\&tlng=es

Organización Mundial de la Salud. (2004). La organización del trabajo y el estrés. Nottingham: OMS.

Parraga, L. (2020). Universidad Técnica de Manabí. Obtenido de Google Forms: https://docs.google.com/form $\mathrm{s} / \mathrm{d} / 1 \mathrm{YULQ}$ -

DdR7JojLqBlajujAg4jrTo07_q UIT4fhdt3ymE/edit\#response $=A C Y D B N j T q h$ -

WyuJrVZ3BbS706PTrjxiaaU wgY9uimbiqRClvGKpqC5rXS zRYB5_PM3DAMns

Ticona, L. (2018). FACTORES PREDOMINANTES QUE INCIDEN EN EL ESTRÉS LABORAL EN COLABORADORES DEL HOSPITAL BASE III. Repositorio de la Universidad Nacional del Altiplano, 1-87. Obtenido de http://repositorio.unap.edu.pe 
/bitstream/handle/UNAP/8218

/Ticona_Mamani_Leydeby_G

aby.pdf?sequence=1\&isAllow

$\mathrm{ed}=\mathrm{y}$

Velásquez, A., \& Escobar , G.

(2020). Estrés Laboral y

síntomas psicosomáticos en

el personal administrativo del Ministerio de Transporte y

Obras Públicas Zona 4

Distrito Portoviejo. Revista

Caribeña de Ciencias

Sociales. Obtenido de https://www.eumed.net/rev/ca ribe/2020/06/estres-

laboral.html

Vidal, V. (2019). El estrés laboral: Análisis y prevención. Zaragoza: Prensas de la Universidad de Zaragoza.

Warr, P., Wall, T., \& Cook, J. (1979). Scales for the Measurement of Some Work Attitudes and Aspects of Psychological Well-Being. Journal of Occupational Psychology, 2(52), 129-148. Obtenido de https://www.researchgate.net/ publication/232573383_Scale s_for_the_Measurement_of_ Some_Work_Attitudes_and Aspects_of_Psychological_W ell-Being 